Cahiers de la recherche sur les droits

Cahiers

sur les Droits fondamentaux

9 | 2011

Conseil constitutionnel et droits fondamentaux

\title{
La terminologie des " droits fondamentaux » dans la jurisprudence du Conseil constitutionnel
}

Samuel Etoa

\section{(2) OpenEdition}

\section{Journals}

Édition électronique

URL : https://journals.openedition.org/crdf/5390

DOI : $10.4000 /$ crdf.5390

ISSN : 2264-1246

Éditeur

Presses universitaires de Caen

Édition imprimée

Date de publication : 1 décembre 2011

Pagination : 23-29

ISBN : 978-2-84133-396-7

ISSN : 1634-8842

Référence électronique

Samuel Etoa, «La terminologie des « droits fondamentaux » dans la jurisprudence du Conseil constitutionnel », Cahiers de la recherche sur les droits fondamentaux [En ligne], 9 | 2011, mis en ligne le 01 décembre 2012, consulté le 14 novembre 2022. URL : http://journals.openedition.org/crdf/5390 ; DOI : https://doi.org/10.4000/crdf.5390 


\title{
La terminologie des "droits fondamentaux " dans la jurisprudence du Conseil constitutionnel
}

\author{
Samuel ETOA \\ Maître de conférences en droit public à l'Université de Caen Basse-Normandie \\ Centre de recherche sur les droits fondamentaux et les évolutions du droit (CRDFED)
}

I. Une terminologie dépourvue de spécificité dans la jurisprudence constitutionnelle
A. La (re)construction de l'objet: le rôle de la doctrine constitutionnaliste
B. Le rejet évident d'une catégorie juridique dans la jurisprudence constitutionnelle

II. Une terminologie non dépourvue d'intérêt dans la jurisprudence constitutionnelle

A. Une terminologie globalement avantageuse: I'approche sémantique des "droits fondamentaux"

B. Une terminologie véritablement opératoire: I'approche fonctionnelle des "droits fondamentaux»

La terminologie des «droits fondamentaux» donne lieu depuis une vingtaine d'années à une pluralité de discours théoriques ${ }^{1}$. En effet, contrairement à ce qui se fait dans nombre d'États européens, la France ne connaît aucun catalogue de ces droits. Certes, on trouve dans le paysage juridique français des textes qui renvoient au vocabulaire de la fondamentalité mais ces derniers ne sont que d'une aide relative. Dans la majorité des cas, le jurislateur garde le silence sur la liste des normes susceptibles de bénéficier de cette appellation et/ou sur la définition du droit ou de la liberté «fondamental(e) $»^{2}$. Aussi la définition de ces prérogatives occupe-t-elle une place centrale chez les juristes français.
On doit rappeler à cet égard qu'une partie non négligeable de l'engouement doctrinal pour les droits fondamentaux provient de l'action du Conseil constitutionnel et de la propension de cette institution à user du vocabulaire de la fondamentalité.

Les étapes de cette consécration sont relativement bien connues. Dans la décision sur les nationalisations, la haute instance consacre «[...] le caractère fondamental» du droit de propriété ${ }^{3}$. Par la suite, la décision des 10 et 11 octobre 1984 relative aux entreprises de presse affirme que la liberté de communication des idées et des opinions proclamée par l'article 11 de la Déclaration des Droits de l'homme et du citoyen du 26 août 1789 est «une liberté

1. Qu'il soit permis sur ce point de renvoyer à notre travail: Le passage des «libertés publiques» aux «droits fondamentaux». Analyse des discours juridiques français, Thèse, Université de Caen Basse-Normandie, juillet 2010.

2. Les exemples du référé «liberté fondamentale» mis en place par la loi du 30 juin 2000 et des projets de 1990 et 1993 qui visaient à l'introduction d'une procédure d'exception d'inconstitutionnalité nous paraissent symptomatiques d'un attrait des pouvoirs publics pour la langue et le discours des droits fondamentaux autant que d'une difficulté de ces derniers à distinguer ce qui relève ou non de cette appellation.

3. Conseil constitutionnel, déc. $\mathrm{n}^{\circ}$ 81-132 DC du 16 janvier 1982, Loi de nationalisation, JORF, 17 janvier 1982, p. 299 , considérant ${ }^{\circ} 16$. 
fondamentale d'autant plus précieuse que son exercice est l'une des garanties essentielles du respect des autres droits et libertés et de la souveraineté nationale ${ }^{4}$. Il faudra cependant attendre la décision $n^{\circ} 89-269$ DC pour que la haute instance utilise l'expression au pluriel. Dans le $33^{\mathrm{e}}$ considérant, le Conseil constitutionnel estime que: « le législateur peut prendre à l'égard des étrangers des mesures spécifiques à condition de respecter les engagements internationaux souscrits par la France et les libertés et droits fondamentaux de valeur constitutionnelle reconnus à tous ceux qui résident sur le territoire de la République ${ }^{5}$.»

La même formule sera reprise dans la décision $M a \hat{\imath}-$ trise de l'immigration ${ }^{6}$. Toutefois, la référence aux droits fondamentaux apparaît dans le $3^{\mathrm{e}}$ considérant dans un paragraphe consacré aux «normes de constitutionnalité applicables à la loi déférée». De plus, le Conseil ne se contente plus d'évoquer brièvement la locution consacrée dans la décision du 22 janvier 1990 mais fournit une liste indicative de droits qu'il considère «fondamentaux ${ }^{7}$. L'appellation générique «droits fondamentaux» se retrouvera par ailleurs avec quelques variations dans des décisions ultérieures ${ }^{8}$. La décision $n^{\circ}$ 98-401 DC marque cependant un tournant puisqu'elle constitue la dernière manifestation du vocable dans la jurisprudence constitutionnelle ${ }^{9}$. Une telle utilisation, à la fois concentrée dans le temps et extrêmement libre dans son maniement, a longtemps posé la question d'une catégorie spécifique de droits dans les décisions du Conseil. Dans un article de référence, MM. Favre et Tardivel s'interrogent sur l'existence et les contours d'une catégorie jurisprudentielle. Et ces derniers de souligner l'influence de la pensée constitutionnaliste dans la réception et l'utilisation de ce vocable par les juges de la constitutionnalité des lois ${ }^{10}$.

La terminologie des droits fondamentaux aurait donc profité d'influences croisées entre la doctrine et l'institution. Certes, mais pouvons-nous ramener le succès de celle-ci à ce seul argument? La réponse est évidemment négative. Rares sont pourtant les auteurs à s'être penchés sur la locution «droits fondamentaux» ou, pour le dire plus clairement, sur les raisons qui ont fondé l'utilisation de celle-ci dans les discours du jurislateur. Pour la doctrine en effet, tout se passe comme si expression et notion étaient inextricablement liées, comme s'il suffisait pour comprendre le recours à cette référence de s'entendre sur ce qui fait qu'un droit est ou, au contraire, n'est pas «fondamental».
Mais il y a parfois plus qu'une nuance entre l'utilisation d'un vocable et l'existence d'un objet juridique particulier. Il arrive fréquemment au juriste ou au jurislateur d'user de mots, d'expressions, faiblement déterminé(e) $s$, pour ne pas dire totalement flous. Dès lors, on ne doit peut-être pas s'étonner si les récentes études qui traitent de l'usage des droits fondamentaux dans la jurisprudence constitutionnelle démontrent l'absence d'une catégorie juridique spécifique. Le générique, un temps employé par le Conseil constitutionnel, ne renvoie, dans les décisions de cette institution, à aucun ensemble particulier, à aucune notion déterminée ou déterminable. C'est dire, par conséquent, que prise sous l'angle du concept juridique, la référence aux droits fondamentaux est dépourvue de spécificité (I), ce qui ne signifie pas pourtant qu'elle soit dénuée d'intérêt (II).

\section{Une terminologie dépourvue de spécificité dans la jurisprudence constitutionnelle}

Les droits fondamentaux ont d'abord été utilisés en doctrine avant d'apparaître dans les décisions du Conseil constitutionnel. L'expression était alors directement dérivée du droit allemand. Ce point est important dans la mesure où le passage de la conception allemande à la conception française des droits fondamentaux suppose qu'un travail approfondi de réappropriation conceptuelle ait été effectué de la part des auteurs (A). S'il est vrai que la terminologie se retrouve ensuite dans la jurisprudence $\mathrm{du}$ Conseil, il s'en faut toutefois beaucoup pour que cette institution confère à l'expression un sens juridique particulier (B).

\section{A. La (re)construction de l'objet: le rôle de la doctrine constitutionnaliste}

Lorsque l'on parle de droits fondamentaux, l'Allemagne fait rapidement office de modèle, de source d'inspiration. Rares sont les auteurs à ne pas rappeler la filiation qui existe entre les droits fondamentaux et les Grundrechte allemands. Le doyen Favoreu écrivait, par exemple, que l'expression n'était « utilisée qu'en Allemagne fédérale où

4. Conseil constitutionnel, déc. $\mathrm{n}^{\circ} 84-181$ DC des 10 et 11 octobre 1984, Entreprises de presse, JORF, 13 octobre 1984, p. 3200, considérant n ${ }^{\circ} 37$.

5. Conseil constitutionnel, déc. $\mathrm{n}^{\circ}$ 89-269 DC du 22 janvier 1990, Loi portant diverses dispositions relatives à la Sécurité sociale et à la santé, JORF, 24 janvier 1992, p. 972, considérant $\mathrm{n}^{\circ} 33$.

6. Conseil constitutionnel, déc. $\mathrm{n}^{\circ}$ 93-325 DC du 13 août 1993, Mâ̂trise de l'immigration, JORF, 18 août 1993, p. 11722.

7. Déc. $\mathrm{n}^{\circ} 93-325 \mathrm{DC}$, considérant $\mathrm{n}^{\circ} 3,81,84$.

8. Conseil constitutionnel, déc. $\mathrm{n}^{\circ}$ 94-345 DC du 29 juillet 1994, Loi relative à l'emploi de la langue française, JORF, 2 août 1994, p. 11240, considérant $\mathrm{n}^{\circ} 5$; déc. $\mathrm{n}^{\circ}$ 97-389 du 22 avril 1997, Loi portant diverses dispositions relatives à l'immigration, JORF, 25 avril 1997, p. 6271; déc. $\mathrm{n}^{\circ}$ 98-401 $\mathrm{DC}$ du 10 juin 1998, Loi d'orientation et d'incitation relative à la réduction du temps de travail, JORF, 14 juin 1998, p. 9033, considérant $\mathrm{n}^{\circ} 3$.

9. Déc. $\mathrm{n}^{\circ} 98-401$.

10. J. Favre et B. Tardivel, «Recherche sur la catégorie jurisprudentielle des "libertés et droits fondamentaux de valeur constitutionnelle" ", RDP, 200o, p. 1415-1418 
elle prend un sens précis prévu par la Constitution ${ }^{11}$. Le propos est ici symptomatique à deux points de vue. Il l'est tout d'abord dans la mesure où le doyen Favoreu se réfère exclusivement à l'Allemagne alors que d'autres États européens usaient à la même époque de cette référence dans leurs constitutions ${ }^{12}$. En outre, les termes de l'auteur d'Aix soulignent l'existence d'un sens précis conféré à l'expression «droits fondamentaux» outre-Rhin. Ces considérations n'empêchent toutefois pas ce dernier d'envisager les droits fondamentaux comme une catégorie générique, une catégorie regroupant l'ensemble des droits et libertés constitutionnellement protégés ${ }^{13}$. Il s'agit là d'une acception relativement large puisqu'elle prend en compte l'ensemble des droits garantis par le contrôle de constitutionnalité des lois, peu importe qu'ils soient ou non inscrits dans le texte constitutionnel ${ }^{14}$.

Mais, cette acception des droits fondamentaux est très différente de celle qui est utilisée outre-Rhin où tous les droits constitutionnels ne sont pas qualifiés de Grundrechte par la Loi fondamentale ${ }^{15}$. Surtout, la qualification de droit fondamental entraîne en Allemagne une protection spécifique caractérisée par le recours de l'article 93 alinéa 4a de la Constitution, recours qui permet à toute personne s'estimant lésée dans l'un de ses droits de saisir la Cour fédérale de Karlsruhe. Une telle garantie n'existe bien évidemment pas en France au début des années 1980. La protection des libertés contre les actes émanés du législateur ne date en effet que des années 1970 et le contrôle opéré par le Conseil constitutionnel n'est effectué qu'entre les phases de vote et de promulgation de la loi, ce qui explique le caractère restrictif des textes relatifs aux autorités de saisine. Rien, par conséquent, de comparable avec l'exemple allemand où les droits fondamentaux sont avant tout pensés comme une catégorie spécifique de droits subjectifs constitutionnels et qualifiés comme tels par la Loi fondamentale ${ }^{16}$.

Dès lors, on peut se demander comment s'est opéré le glissement des Grundrechte aux droits fondamentaux. Il faut, pour comprendre ce point, se référer à l'article que Michel Fromont publie en 1975 dans les Mélanges Eisenmann ${ }^{17}$. Dans cet article de référence, les droits fondamentaux sont appréhendés plutôt dans leur signification objective que subjective. Cette transformation dans la manière d'appréhender l'objet n'est pas sans conséquence sur la notion puisqu'elle tend à mettre en valeur le caractère structurant des droits fondamentaux. Or, ce caractère tient avant tout à la place prééminente qu'occupent ces droits dans l'ordre juridique allemand. Le contrôle de constitutionnalité exercé par la Cour fédérale prend bien sûr un relief particulier mais uniquement en ce qu'il permet la subordination effective de l'ensemble des pouvoirs publics et des différentes branches du droit aux «droits fondamentaux». Et si l'auteur évoque l'existence du recours constitutionnel dans cet article, on ne doit pas oublier que ce qui est prioritairement décrit demeure la capacité des droits fondamentaux à ensemencer l'ordre juridique, capacité qui résulte moins du caractère fondamental ou de la protection spécifique qui leur est conféré(e) que de leur statut de normes constitutionnelles et/ou de droits constitutionnellement protégés.

C'est l'apanage du doyen Favoreu que d'avoir démontré l'existence d'une problématique de constitutionnalisation des branches du Droit ${ }^{18}$, comparable à celle qui se donnait à voir en Allemagne, chaque fois qu'existent dans un ordre juridique donné, un stock de normes constitutionnelles de fond et une procédure de contrôle matériel de la constitutionnalité des lois, fût-elle exercée à titre préventif ${ }^{19}$. Des Grundrechte aux droits fondamentaux, c'est donc l'idée d'une transformation de l'objet initial qui se fait jour dans les travaux de la doctrine néoconstitutionnaliste. Et si l'expression s'est rapidement retrouvée dans la jurisprudence constitutionnelle, force est néanmoins de constater que cette consécration ne nous renvoie pas à un objet stable et précis.

\section{B. Le rejet évident d'une catégorie juridique dans la jurisprudence constitutionnelle}

L'apparition des droits fondamentaux dans la jurisprudence du Conseil constitutionnel a été interprétée comme le signe d'une sensibilité de cette institution au langage de la doctrine. De là à penser qu'il y a derrière l'usage de cette terminologie un renvoi aux prescriptions de la pensée néo-constitutionnaliste, il n'y a qu'un pas que certains ont aisément franchi. À aucun moment pourtant le Conseil constitutionnel n'a consacré le principe d'une catégorie de «droits fondamentaux» qui regrouperait l'intégralité des droits et libertés constitutionnellement protégés. Tout au contraire. Ainsi, la haute instance réserve-t-elle cette qualification à un petit nombre de droits dotés de la protection constitutionnelle, voire à certains éléments

11. L. Favoreu, «Rapport général introductif», in Cours constitutionnelles européennes et droits fondamentaux, L. Favoreu (dir.), Paris - Aix-enProvence, Economica - Presses universitaires d'Aix-Marseille, 1982, p. 41.

12. Ainsi de la Constitution portugaise et de la Constitution espagnole.

13. Ibid.

14. Ibid.

15. Ainsi, la liberté de constituer des partis politiques consacrée à l’article 21 alinéa 1 de la Loi fondamentale n'appartient ni à la liste des droits fondamentaux ni à celle des droits identiques ou assimilables à des droits fondamentaux de l'article 93 alinéa $4 \mathrm{a}$.

16. D. Capitant, Les Effets juridiques des droits fondamentaux en Allemagne, Paris, LGDJ (Bibliothèque constitutionnelle et de science politique, t. 87$), 2001$, p. 3

17. M. Fromont, «Les droits fondamentaux dans l'ordre juridique de la République fédérale d'Allemagne», Recueil d'études en hommage à Charles Eisenmann, Paris, Cujas, 1975, p. 49-64.

18. L. Favoreu, «La constitutionnalisation du droit», in L'unité du droit. Mélanges en hommage à Roland Drago, Paris, Economica, 1996 , p. $25-42$.

19. L. Favoreu et T. S. Renoux, "Rapport général introductif», La Cour de cassation et la Constitution de la République, Actes du colloque des 9 et 10 décembre 1994 organisé par le GERJC et la Cour de cassation à Paris, Aix-en-Provence, Presses universitaires d'Aix-Marseille, 1995, p. 20. 
seulement de ces prérogatives. Dans la décision sur les nationalisations, le juge distingue l'impératif de préservation du droit de propriété des garanties qui s'y attachent. Il ne qualifie finalement de «fondamental» que le premier de ces aspects ${ }^{20}$. Dans le domaine de la liberté de communication, le Conseil constitutionnel a été amené à dissocier la liberté proclamée par l'article 11 de la Déclaration des Droits de l'homme et du citoyen de 1789 de la qualification de droit ou de liberté fondamental(e). Ainsi la liberté de l'article 11 de la Déclaration n'est-elle une liberté fondamentale que par intermittence. Elle l'est, par exemple, dans le cadre général de la décision sur les entreprises de presse mais cesse de l'être lorsqu'est en cause la communication audiovisuelle ${ }^{21}$. On voit mal dans ces conditions comment conserver la possibilité d'une catégorie générique fondée sur la protection constitutionnelle du droit et/ou de la liberté en cause. Une approche aussi stricte de la fondamentalité était sans doute trop embarrassante pour le Conseil constitutionnel qui étend la liste des droits et libertés constitutionnellement protégés bien au-delà du cadre de la Constitution écrite.

Ce constat néanmoins a permis que soit discutée en doctrine l'hypothèse d'une catégorie spécifique. Pour certains, les droits fondamentaux se caractériseraient par l'existence d'un régime juridique renforcé. Toutefois, les membres de la doctrine ne s'accordent pas sur les traits de ce régime, ni d'ailleurs sur la liste des droits qui en bénéficient ${ }^{22}$. En outre, le Conseil constitutionnel n'applique pas les éléments de ce régime renforcé à tous les droits qu'il considère comme étant "fondamentaux» ${ }^{23}$. Si l'on ajoute à cela que le principe du cliquet anti-retour - principe qui était par ailleurs le seul à se retrouver constamment dans les recherches doctrinales sur le sujet - n'est plus utilisé depuis plusieurs années maintenant dans la jurisprudence constitutionnelle ${ }^{24}$, on comprend que cette catégorie de droits ne peut se définir par un régime juridique qui serait distinct des autres droits et libertés constitutionnellement protégés.

Enfin, une autre définition spécifique des droits fondamentaux s'est développée sous l'impulsion d'Étienne Picard ${ }^{25}$. Selon cette approche, la fondamentalité ne serait liée ni à la norme ni au niveau de protection du droit considéré mais serait inhérente à la substance de la prérogative en question. Les droits fondamentaux seraient ainsi des droits prééminents puisque attachés au principe de dignité de la personne humaine ${ }^{26}$. Une telle acception qui a le mérite de poser une alternative aux conceptions par trop positivistes de la théorie normativiste est cependant écartée dans la jurisprudence constitutionnelle. Il suffit, en effet, de parcourir les décisions du Conseil pour constater que la qualification de droit et/ou de liberté fondamental(e) n'est attachée ni à l'humanité du titulaire de la prérogative ni à la sauvegarde de la dignité de ce dernier.

Ainsi, les personnes morales jouissent-elles dans les décisions du Conseil constitutionnel de droits expressément qualifiés de fondamentaux. Dans la décision $n^{\circ}$ 93-325, par exemple, le Conseil constitutionnel reconnaît le caractère fondamental des droits de la défense, droits qui, selon les mots de la haute instance, bénéficient à «toutes les personnes» ${ }^{27}$. De même, dans la décision $n^{\circ}$ 94-345 sur l'emploi de la langue française, le juge constitutionnel qualifie la liberté de communication de liberté fondamentale avant de reconnaître que le législateur ne pouvait, pour cette raison, imposer sous peine de sanctions certaines obligations aux «organismes et services de radiodiffusion sonore et télévisuelle qu'ils soient publics ou privés ${ }^{28}$. À l'inverse, le juge constitutionnel n'a pas attribué cette qualité à la dignité de la personne humaine ${ }^{29}$, principe qui, au regard des postulats substantialistes, aurait amplement mérité cette dénomination. La référence aux droits fondamentaux s'avère ainsi rétive à toute tentative de systématisation conceptuelle. Mais cela ne signifie pas qu'elle soit dépourvue d'intérêt.

\section{Une terminologie non dépourvue d'intérêt dans la jurisprudence constitutionnelle}

Dans la mesure où aucun des critères d'explicitation posés par la doctrine ne permet d'appréhender ce qui, dans la jurisprudence constitutionnelle, est «droit» ou «liberté»

20. Déc. $\mathrm{n}^{\circ} 81-132$, considérant $\mathrm{n}^{\circ} 16$.

21. Comparer déc. $\mathrm{n}^{\circ}$ 84-181 DC, considérant $\mathrm{n}^{\circ} 37$, et déc. $\mathrm{n}^{\circ}$ 86-217 DC du 18 septembre 1986, Loi relative à la liberté de communication audiovisuelle, JORF, 19 septembre 1986, p. 11294.

22. T. Meindl, La notion de droit fondamental dans les jurisprudences et doctrines constitutionnelles françaises et allemandes, Paris, LGDJ (Bibliothèque constitutionnelle et de science politique, t. 112), 2003, p. 72-73.

23. Le droit de propriété dont le caractère fondamental a pourtant été souligné par le Conseil constitutionnel ne bénéficie pas de cette protection renforcée, déc. $\mathrm{n}^{\circ}$ 81-132 DC.

24. Sur ce point, J.-E. Schoettl, «La nouvelle loi relative au droit d'asile est-elle constitutionnelle?», LPA, n $26,4-5$ février 2004, p. 12-13; Conseil constitutionnel, déc. $\mathrm{n}^{0}$ 2009-580 DC du 10 juin 2009, Loi $n^{\circ}$ 2009-669 favorisant la diffusion et la protection de la création sur Internet, JORF, 13 juin 2009, considérant $\mathrm{n}^{\circ}$ 15. Dans cette décision qui concernait la liberté de communication, le Conseil constitutionnel ne reprend pas la technique du cliquet anti-retour pourtant présente dans les déc. $\mathrm{n}^{\circ}$ 84-181 DC et 94-345 DC. Le juge se contente en l'espèce d'affirmer que: « Les atteintes portées à l'exercice de cette liberté doivent être nécessaires, adaptées et proportionnées à l'objectif poursuivi (l'objectif de lutte contre les pratiques de contrefaçon sur Internet).»

25. E. Picard, "L'émergence des droits fondamentaux en France», L'Actualité juridique. Droit administratif, $\mathrm{n}^{\circ}$ spécial, Les droits fondamentaux: une nouvelle catégorie juridique?, 1998, p. 6-42.

26. Ibid., p. 32 .

27. Déc. $\mathrm{n}^{\circ} 93-325 \mathrm{DC}$, considérant $\mathrm{n}^{\circ} 84$.

28. Déc. $\mathrm{n}^{\circ} 94-345$ DC, considérant $\mathrm{n}^{\circ} 9$.

29. Conseil constitutionnel, déc. no 94-343-344. 
«fondamental(e)», la question se pose de savoir pourquoi le juge use de cette référence. Deux pistes peuvent alors être exploitées. La première consiste à souligner les avantages qui s'attachent à cette terminologie. Nous qualifierons cette approche de sémantique $(\mathrm{A})$. La seconde, déjà explorée par Véronique Champeil-Desplats, considère les droits fondamentaux comme un outil rhétorique entre les mains du Conseil constitutionnel: c'est l'approche «fonctionnelle» (B).

\section{A. Une terminologie globalement avantageuse: I'approche sémantique des "droits fondamentaux"}

L'utilisation d'un vocable nouveau dans un ordre juridique donné n'est jamais le fruit du hasard. C'est du moins ce qu'explique Étienne Picard dans l'article qu'il publie en 1998 dans le numéro spécial de L'Actualité juridique. Droit administratif ${ }^{30}$. L'apparition des droits fondamentaux dans la jurisprudence constitutionnelle est donc étroitement dépendante des vicissitudes qui affectent les terminologies concurrentes, vicissitudes que la référence aux droits fondamentaux permet justement de dépasser. Deux expressions souffrent ainsi de difficultés qui expliquent qu'elles soient peu employées par le Conseil.

La première est celle des libertés publiques. Bien que largement utilisée dans les discours français, cette terminologie laisse place à certaines imprécisions qui en affectent immédiatement la portée. Sur un plan matériel, tout d'abord, la notion de libertés publiques s'avère incapable de rendre compte de l'évolution des droits. Centrées sur la référence aux libertés et non sur celle des droits, les libertés publiques renvoient à l'idée de facultés d'agir, de pouvoirs d'autodétermination, bref de prérogatives, dont la satisfaction passe par une abstention des sujets passifs. Or, une telle vision implique une césure parmi les Droits de l'homme au détriment des droits-créances, catégorie dont la mise en œuvre renvoie à l'idée de prestation. Cette approche, qui pouvait être compréhensible dans les années 1950, paraît désormais anachronique. Le principe d'une indivisibilité des droits d'abord vanté dans les documents internationaux ${ }^{31}$ et la jurisprudence européenne $^{32}$ a fait son chemin dans la pensée juridique française et les décisions du Conseil constitutionnel. Des obligations positives sont issues de dispositions relatives aux libertés ${ }^{33}$ et des droits-libertés engendrés de dispositions qui concernent les droits-créances ${ }^{34}$. Parler de droits fondamentaux et non de libertés publiques serait ainsi le moyen de dépasser le clivage entre droits-libertés et droitscréances, de marquer l'inanité d'une opposition entre obligation de non-entrave et obligation de prestation. "L'intérêt du terme "droits", écrit le doyen Roussillon, est qu'il a une valeur englobante: si les libertés constituent des "droits", il est des droits qui ne se ramènent pas tous à des "libertés" ${ }^{35}$. Une opinion similaire est défendue par Jean Rivero qui définit le «droit» comme: « un pouvoir reconnu par la Société à un sujet déterminé, d'imposer aux autres certaines obligations, soit négatives - s'abstenir de s'immiscer dans la sphère de liberté propre à chacun -, soit positives: fournir des prestations et des services à celui qui est fondé à les réclamer ${ }^{36}$.»

De «la notion de libertés publiques à celle des droits fondamentaux », c'est donc bien «l'espace normatif» qui s'élargit ${ }^{37}$.

Par ailleurs, on ne peut ignorer le hiatus qui existe entre la terminologie des libertés publiques d'une part et la définition de ces libertés telle qu'elle se fait dans la doctrine majoritaire d'autre part. En effet, les auteurs n'accordent pas à l'adjectif "public» de réelle spécificité. La liberté est dite "publique» chaque fois qu'elle est reconnue par le droit. L'adjectif qui ne fait que renvoyer à une consécration par les pouvoirs publics ne doit donc pas être pris dans le sens d'une coexistence des libertés publiques et des libertés privées. Il est vrai que les quelques auteurs qui soutiennent le principe de libertés privées ne parviennent pas à se mettre d'accord sur les contours de cette catégorie, ni du reste, sur les critères de cette différenciation: s'agit-il avec Dominique Turpin d'évoquer l'opposabilité des libertés aux seules relations privées ${ }^{38}$ ou s'agit-il, comme l'écrivent Jacques Robert et Jean Duffar, d'une question liée à l'attribution de ces libertés ${ }^{39}$ ? Les deux approches sont soutenues et soutenables. Mais elles sont aussi contestables et contestées ${ }^{40}$. Il est difficile pourtant

30. E. Picard, «L'émergence des droits fondamentaux en France», p. 6.

31. Proclamation de Téhéran, 13 mai 1968, ONU, A/Conf., 32/41, \$13; Proclamation de Vienne, 25 juin 1993, A/Conf., 157/23, \$5.

32. Parmi les exemples, CEDH, 9 octobre 1979, Airey c. Irlande, série A, $\mathrm{n}^{\circ} 32 ; \mathrm{CEDH}, 13$ juin 1979, Marcks c. Belgique, série A, $\mathrm{n}^{\circ} 31 . \mathrm{Notons}$ que l'idée d'une indivisibilité des droits a été récemment proclamée en droit communautaire, via le préambule de la Charte des droits fondamentaux de l'Union européenne qui affirme «les valeurs indivisibles et universelles de dignité et de solidarité», valeurs qui constituent selon le texte «le patrimoine spirituel et moral de l'Union». Sur ce texte, G. Braibant, La Charte des droits fondamentaux de l'Union européenne, Paris, Point (Inédit / Essais), 2001.

33. Conseil constitutionnel, déc. $\mathrm{n}^{\circ}$ 86-217 DC, considérant $\mathrm{n}^{\circ} 36$

34. Conseil constitutionnel, déc. $\mathrm{n}^{\circ}$ 93-325 DC, considérants $\mathrm{n}^{\circ}$ 69-70.

35. H. Roussillon, Le Conseil constitutionnel, Paris, Dalloz (Connaissance du droit), 200o, p. 52.

36. J. Rivero, "Science du droit et Droits de l'homme», in Pour les Droits de l'homme. Mélanges en l'honneur de l'Association pour le développement des libertés fondamentales, M. Aubert et al. (dir.), Choisy-le-Roi, Librairie des libertés (Bibliothèque des Droits de l'homme et des libertés fondamentales), 1983 , p. 108.

37. J. Fialaire et E. Mondielli, Droits fondamentaux et libertés publiques, Paris, Ellipses (Université droit), 2005, p. 17.

38. D. Turpin, Libertés publiques et droits fondamentaux, Paris, Seuil, 2004, p. 12.

39. J. Robert et J. Duffar, Droits de l'homme et libertés fondamentales, Paris, Montchrestien (Domat droit public), 1996, p. 14-15.

40. J. Rivero et H. Moutouh, Libertés publiques, $9^{\mathrm{e}}$ édition, Paris, PUF (Thémis droit public), 2003, p. 7 ; P. Wachsmann, Libertés publiques, $6^{\mathrm{e}}$ édition, Paris, Dalloz (Cours), 2009, p. 2; J.-P. Théron, L'essentiel des libertés publiques, $1^{\mathrm{re}}$ édition, Paris, L'Hermès (Droit public), 1994, p. 7. 
de ne pas considérer que l'existence de libertés privées est posée dès que l'on parle de libertés publiques ${ }^{41}$.

Des critiques sont également apparues à l'encontre des Droits de l'homme $e^{42}$. On reproche alors à tort au vocable de renvoyer à des théories jusnaturalistes aujourd'hui dépassées. Les Droits de l'homme ne seraient pas vraiment des droits, mais des prétentions morales dépourvues de force ${ }^{43}$. En outre, la référence à l'homme est jugée trop sexiste $^{44}$. Cette dernière assertion explique, par ailleurs, la tentative de promouvoir des expressions telles que droits de la personne ou droits de la personne humaine. Ces deux expressions demeurent pourtant assez peu présentes dans le vocabulaire juridique français et dans le langage doctrinal qui ne l'emploie qu'à de très rares occasions. Il faut dire que ces terminologies n'ont été reçues en France que récemment. De plus, on peut souligner à propos du générique «droits de la personne humaine » qu'il tend, à l'instar des Droits de l'homme, à ne prendre en compte que les prérogatives dévolues aux individus / personnes physiques et à laisser de côté les droits reconnus aux groupes personnalisés. La question des droits spécifiquement dévolus à des personnes morales de droit privé ou de droit public est, du reste, très présente dans la jurisprudence constitutionnelle française qui reconnaît, dans le sillage des projets d'exception d'inconstitutionnalité des années 1990 et 1993, le bénéfice de droits fondamentaux à l'ensemble des personnes juridiques et non point aux seuls individus, personnes humaines et/ou personnes physiques. Reste alors l'expression «droits de la personne». Le terme «personne» paraît suffisamment large pour englober les droits des personnes physiques et ceux des personnes morales. Mais, l'expression est aussi trop vaste puisque tout droit est, en principe, attribué à une personne juridique. Aussi bien la référence aux «droits fondamentaux», qui implique un choix entre les droits, est-elle plus naturelle.

\section{B. Une terminologie véritablement opératoire: l'approche fonctionnelle des "droits fondamentaux"}

La référence aux droits fondamentaux n'est pas seulement capable de dépasser les critiques qui sont opposées aux autres terminologies, elle est aussi et surtout pour le
Conseil constitutionnel une expression dotée d'un fort pouvoir d'attractivité. C'est ce qu'explique Véronique Champeil-Desplats qui voit dans la référence aux droits fondamentaux un outil rhétorique: un moyen privilégié d'affirmer la légitimité de l'action et des décisions du juge constitutionnel français ${ }^{45}$. À défaut de constituer une véritable catégorie juridique, les droits fondamentaux rempliraient dans la jurisprudence du Conseil un rôle purement argumentatif et légitimant. Cette terminologie était en outre d'autant plus importante aux yeux du Conseil qu'elle intervenait dans un contexte où il importait de «trouver des concepts transnationaux opératoires ${ }^{46}$. La référence aux droits fondamentaux a sans doute été de ces concepts. On constate ainsi que de très nombreux emprunts à la jurisprudence de la Cour de justice de l'Union européenne (ex-CJCE), mais aussi à celle de la Cour européenne des Droits de l'homme, ont émaillé les décisions du Conseil constitutionnel. Des emprunts terminologiques tout d'abord. Car à défaut d'être couramment employée par la Cour européenne des Droits de l'homme, l'expression "droits fondamentaux" était en revanche extrêmement présente dans les discours du droit communautaire et le vocabulaire de la Cour de justice de l'Union européenne qui l'utilisaient depuis la fin des années 1960 et le début des années $1970{ }^{47}$. Des emprunts conceptuels ensuite puisqu'il suffit de croiser la jurisprudence du Conseil constitutionnel et de la Cour européenne des Droits de l'homme pour constater qu'une même volonté d'effectivité des droits anime les institutions ${ }^{48}$.

Reste que le Conseil n'a jamais fait état d'une véritable volonté de systématisation. Non seulement le maniement de l'expression s'avère extrêmement libre chez le juge constitutionnel, mais il semble que les raisons qui ont poussé ce dernier à user du vocabulaire de la fondamentalité soient, elles aussi, relativement diffuses. Tel est le constat d'Étienne Picard qui souligne qu'entre la décision du 16 janvier 1982 et celle des 10 et 11 octobre 1984 sur les entreprises de presse, les causes de la fondamentalité ont permuté. Dans la première, le caractère fondamental semble souligner la valeur constitutionnelle du droit de propriété. L'opération de qualification est alors importante dans la mesure où, selon l'auteur, on doutait à cette époque de la constitutionnalité de ce droit a fortiori lorsqu'il était confronté au principe des nationalisations $^{49}$. Dans la seconde décision, celle sur les entreprises

41. J. Robert et J. Duffar, Droits de l'homme..., p. 14

42. V. Champeil-Desplats, «L'affirmation des droits fondamentaux: quelles significations? Quelles conséquences?», Cahiers français. Liberté/Libertés, $\mathrm{n}^{\circ} 354$, janvier-février 2010, p. 19-23.

43. G. Lebreton, Libertés publiques et Droits de l'homme, $7^{e}$ édition, Paris, Armand Colin (Collection U), 2005, p. 20.

44. G. Koubi, «Droits de l'homme et droits de la personne: réflexion sur l'imprudence d'une distinction", Revue internationale de psychologie, vol. VI, $\mathrm{n}^{\circ} 15,2000$, p. 38.

45. V. Champeil-Desplats, "La notion de droit "fondamental" et le droit constitutionnel français», D., 1995, Chron., p. 323-329.

46. M.-L. Pavia, «Éléments de réflexion sur la notion de droit fondamental», $L P A, \mathrm{n}^{\circ}$ 54, 6 mai 1994, p. 9.

47. CJCE, 12 novembre 1969, Éric Stauder c. ville d'Ulm, affaire 29-69, Rec. CJCE, p. 419; CJCE, 17 décembre 1970, Internationale Handelsgesellschaft, affaire 11/70, Rec. CJCE, p. 1128; CJCE, 14 mai 1974, Nold, affaire 4/73, Rec. CJCE, p. 491.

48. Comparer Conseil constitutionnel, déc. $\mathrm{n}^{\circ}$ 84-181 DC, considérants $\mathrm{n}^{\circ}$ 37-38; CEDH, 26 avril 1979, Sunday Times c. Royaume-Uni, série A, ${ }^{\circ}$ 30, $\$ 65 ; \mathrm{CEDH}, 7$ septembre 1976, Handyside c. Royaume-Uni, série A, nº 24, $\$ 49$.

49. E. Picard, «L'émergence des droits fondamentaux en France», p. 11. 
de presse, «c'est plutôt la qualité constitutionnelle qui justifie la fondamentalité ${ }^{50}$.

Le Conseil constitutionnel use, par conséquent, de la qualification de fondamentalité selon l'effet désiré: souligner la constitutionnalité d'un droit ou marquer la place particulière de tel(le) principe ou prérogative parmi les éléments du bloc de constitutionnalité lorsque le juge est confronté à un conflit de normes. Le fait que le juge constitutionnel ait décidé depuis la décision du 10 juin 1998 de ne plus recourir à cette qualification dans le cadre de ses décisions signifie bien que les droits fondamentaux ne sont rien d'autre qu'une expression commode, mobilisable au gré des envies de ce dernier. Il est du reste symptomatique de constater que la théorie fonctionnelle un temps controversée soit désormais celle qui explique de la manière la plus évidente les occurrences du recours aux droits fondamentaux dans les décisions du Conseil. Les auteurs ne s'y trompent d'ailleurs pas qui du professeur Patrick Wachsmann ${ }^{51}$, d'Olivier Le Bot ${ }^{52}$ ou d'Olivier Dord ${ }^{53}$ ont explicitement pris fait et cause en faveur de celle-ci. Quant à connaître les raisons qui ont conduit le juge constitutionnel à délaisser cette rhétorique, nous ne pouvons formuler que des hypothèses. Le peu d'intérêt que semblent éprouver les constituants pour les droits fondamentaux peut expliquer en partie cet abandon. Certes, les projets d'exception d'inconstitutionnalité des lois de 1990 et 1993 reprenaient cette terminologie. Plus récemment, la proposition $\mathrm{n}^{\circ} 74$ qui préfigurait la procédure de la question prioritaire de constitutionnalité affirmait que «Le Conseil constitutionnel peut, à l'occasion d'une instance en cours devant une juridiction, être saisi par voie d'exception aux fins d'apprécier la conformité d'une loi aux libertés et droits fondamentaux reconnus par la Constitution ${ }^{54}$ ».

Dont acte... Nous constaterons cependant que les projets de 1990 et 1993 n'ont jamais vu le jour et que la référence aux droits fondamentaux est aujourd'hui absente du texte de l'article 61-1 qui préfère évoquer les «droits et libertés que la Constitution garantit ${ }^{55}$. Toutes choses qui semblent finalement avoir sonné le glas de cette référence dans la jurisprudence constitutionnelle française...

50. Ibid., p. 12

51. P. Wachsmann, "L'importation en France de la notion de "droits fondamentaux" ", RUDH, 2004, p. 46

52. O. Le Bot, La protection des libertés fondamentales par la procédure du référé-liberté. Étude de l'article L. 521-2 du Code de justice administrative, Paris, LGDJ - Fondation Varenne (Thèse, $\mathrm{n}^{\circ}$ 9), 2007, p. 68.

53. O. Dord, «Droits fondamentaux», in Dictionnaire des droits fondamentaux, J. Andriantsimbazovina, H. Gaudin, J.-P. Marguénaud, S. Rials et F. Sudre (dir.), Paris, PUF (Quadrige / Dicos Poche), 2008, p. 333

54. Comité de réflexion et de proposition sur la modernisation et le rééquilibrage des institutions de la $V^{e}$ République, Pour une Ve République plus démocratique, p. 90.

55. L'article 61-1 de la Constitution se réfère uniquement aux «droits et libertés que la Constitution garantit». 
